\title{
Oral contraceptive pill use and the susceptibility to markers of exercise-induced muscle damage
}

\author{
K. M. Hicks ${ }^{1,2} \cdot$ G. Onambélé-Pearson ${ }^{2} \cdot$ K. Winwood ${ }^{2}$ C. I. Morse ${ }^{2}$
}

Received: 23 November 2016 / Accepted: 1 May 2017 / Published online: 11 May 2017

(C) The Author(s) 2017. This article is an open access publication

\begin{abstract}
Purpose Firstly, to establish whether oral contraceptive pill (OCP) users are more susceptible to muscle damage compared to non-users, and secondly, to establish whether differences can be attributed to differences in patella tendon properties.

Methods Nine female OCP users and 9 female non-users participated in the investigation. Combining dynamometry, electromyography and ultrasonography, patella tendon properties and vastus lateralis architectural properties were measured pre and during the first of 6 sets of 12 maximal voluntary eccentric knee extensions. Serum oestrogen levels were measured on the 7th day of the pill cycle and the 14th day of menstrual cycle in OCP users and non-users, respectively. Maximal voluntary isometric knee extension torque loss, creatine kinase and muscle soreness were measured $48 \mathrm{~h}$ pre-damage, post-damage, and 48, 96 and $168 \mathrm{~h}$ post-damage.

Results Oestrogen levels were significantly lower in OCP users compared to non-users $(209 \pm 115$ and $433 \pm 147 \mathrm{pg} / \mathrm{ml}$, respectively, $p=0.004)$. Proposed determinants of muscle damage, patella tendon stiffness and maximal eccentric torque did not differ between OCP users
\end{abstract}

Communicated by Anni Vanhatalo.

K. M. Hicks

Kirsty.Hicks@northumbria.ac.uk

1 Department of Sport, Exercise and Rehabilitation, Northumbria University, Newcastle City Campus, 2 Ellison Place, Newcastle upon Tyne NE1 8ST, UK

2 Health Exercise and Active Living Research Centre, Exercise and Sport Science, Manchester Metropolitan University, Crewe CW1 5DU, UK and non-users. The change in creatine kinase from pre to peak was significantly higher in OCP users compared to non-users $(962 \pm 968$ and $386 \pm 474 \mathrm{Ul}$, respectively, $p=0.016$ ). There were no other differences in markers of muscle damage.

Conclusion Although our findings suggest that, when compared to non-users, the OCP may augment the creatine kinase response following eccentric exercise, it does not increase the susceptibility to any other markers of muscle damage.

Keywords Exercise-induced muscle damage $\cdot$ Oral contraceptive pill $\cdot$ Tendon properties
Abbreviations
ANOVA Analysis of variance
CK Creatine kinase
EIMD Exercise-induced muscle damage
$\mathrm{MVE}_{\mathrm{KE}} \quad$ Maximal voluntary eccentric knee contractions
$\mathrm{MVC}_{\mathrm{KE}}$ Maximal voluntary isometric knee extension
$\mathrm{MVC}_{\mathrm{KF}} \quad$ Maximal voluntary isometric knee flexions
OCP Oral contraceptive pill
$\Delta \mathrm{CK}_{\text {peak }}$ The increase from pre to peak CK over $168 \mathrm{~h}$
VL Vastus lateralis
$\mathrm{VL}_{\mathrm{ACSA}} \quad$ Vastus lateralis anatomical cross-sectional area

\section{Introduction}

Exercise-induced muscle damage (EIMD) has been shown to be lower in females compared to males (Joyce et al. 2014; Sewright et al. 2008). It has been suggested that this attenuated EIMD is due to the direct antioxidant properties of oestrogen (Carter et al. 2001). However, an indirect role of oestrogen in suppressing EIMD, through altering the 
tendon properties in females, has recently been proposed (Hicks et al. 2016).

The role of oestrogen in attenuating EIMD has been assessed through the manipulation of circulating levels in animals and has been shown to attenuate EIMD in both male and female rats (Amelink et al. 1990, 1991; Bär et al. 1988). For example, Bär et al. (1988) reported that the increase in creatine kinase (CK) post-EIMD, in both males and ovariectomised female rats, was suppressed by oestrogen supplements prior to EIMD. Alternatively, within human studies, oestrogen levels can be manipulated through the use of synthetic hormones [e.g. hormone replacement therapy and the oral contraceptive pill (OCP)].

The OCP down regulates oestrogen levels throughout the menstrual cycle by altering the hypothalamic-pituitary-ovarian feedback loop, thus inhibiting the peak in oestrogen at ovulation (Elliott-Sale et al. 2013; Van Heusden and Fauser 2002). Consequently, women who take the OCP have significantly lower levels of circulating oestrogen compared to non-users throughout the menstrual cycle (Bryant et al. 2008; Fleischman et al. 2010). Due to the protective properties of oestrogen and the suppressed oestrogen levels in OCP users, it is suggested that OCP users are more susceptible to EIMD; however, the literature remains inconclusive with evidence supporting (Carter et al. 2001; Joyce et al. 2014; Minahan et al. 2015) or refuting (Sewright et al. 2008; Thompson et al. 1997) an increased susceptibility to EIMD. For example, in support of an increased susceptibility to EIMD, 48-h post-maximal eccentric leg extensions Joyce et al. (2014), reported significantly higher levels of serum CK levels in males and female OCP users compared to female non-users. Whereas, following maximal eccentric contractions of the elbow flexors, Sewright et al. (2008) reported no significant difference in CK between OCP users and non-users. The differences in exercise protocols/exercising muscle groups (Chen et al. 2011) and different phases of the menstrual cycle/oestrogen levels, make it difficult to determine the true role of the OCP on EIMD.

In addition to acting as an antioxidant, oestrogen may indirectly attenuate EIMD through altering tendon properties. Compliant tendons have been evidenced to mitigate fascicle lengthening (Hicks et al. 2013) and attenuating peak forces and torques during eccentric contractions (Hoffman et al. 2014; Roberts and Azizi 2010; Roberts and Konow 2013). Therefore, it is suggested that lower tendon stiffness may act as a mechanical buffer and attenuate levels of EIMD (Hoffman et al. 2014; Roberts and Azizi 2010; Roberts and Konow 2013).

High oestrogen levels have been reported to decrease tendon stiffness by attenuating the rate of tendon collagen synthesis (Hansen et al. 2009a, b). In parallel, IGF-I has an anabolic effect on tendon fibroblasts by increasing collagen syntheses in a dose-dependent manner (Olesen et al. 2006) and hence would be assumed to be associated with higher tendon stiffness. Interestingly, oestrogen is associated with decreased IGF-I bioavailability (Heald et al. 2005). In other words, a lower tendon stiffness would be expected in the presence of elevated oestrogen due to the sequestering away of IGF. Within OCP users, however, despite lower oestrogen levels compared to non-users, it remains unclear, with arguments supporting (Bryant et al. 2008) or contesting (Hansen et al. 2013) differences in tendon properties between OCP users and non-users. It is possible, however, that the aforementioned discrepancies between Bryant et al. (2008) and Hansen et al. (2013) findings may be attributed to differences in the number of available oestrogen receptors expressed on the Achilles and patella tendon.

Therefore, the aim of the current study was twofold: firstly, to establish whether OCP users are more susceptible to EIMD compared to non-users and secondly, to establish whether any differences in EIMD could be attributed, in part, to differences in tendon properties.

\section{Materials and methods}

\section{Participants}

A total of 18 females were divided into two groups: women taking a monophasic OCP $(n=9,23.4 \pm 2.4$ years of age, $70.0 \pm 9.9 \mathrm{~kg}$ and $1.70 \pm 0.05 \mathrm{~m}$ ) and eumenorrheic women who had never taken the OCP [(non-users) $n=9$, $21.2 \pm 1.5$ years of age, $63.2 \pm 5.6 \mathrm{~kg}$ and $1.65 \pm 0.09 \mathrm{~m}]$. All participants self-reported as being recreationally active (undertaking no more than $1 \mathrm{~h}$ of "moderate" physical activity per week) and did not take part in any structured resistance training. All procedures complied with the Declaration of Helsinki World Medical Association (2013) and ethical approval was obtained through the local ethics committee at Manchester Metropolitan University. All women reported regular menstrual cycles, documenting an average cycle length of $28 \pm 1$ days. On average the OCP users had been taking a combined monophasic OCP, with an ethinyl estradiol dosage between 20 and $30 \mu$ for $3 \pm 1$ years. The types of OCP used within the current study are taken daily followed by a 7-day pill-free interval. Exclusion criteria included any resistance training undertaken in the last 6 months, occupation or lifestyle that required regular heavy lifting or carrying, any known muscle disorder, the use of dietary supplements (for example, multivitamins), and any musculoskeletal injury in the last 3 months. Further exclusion criteria included, previous use of any other forms of hormone-based contraception, continuous pill OCP or mini-OCP users, irregular menstrual cycles [where regular cycles were defined as $24-36$ days (Cole et al. 
2009; Landgren et al. 1980)] in the last 12 months, and pregnancy in the year preceding inclusion in the current study. All inclusion and exclusion criteria were determined through participant questionnaire prior to inclusion within this investigation.

\section{Testing protocol}

Once selected, participants were asked to visit the laboratory on five different occasions over 9 days. Non-users were tested on the 14th day (self-reported) of the menstrual cycle to measure oestrogen levels at ovulation (Brown 1955; Cole et al. 2009). To reflect the timing of ovulation in the non-users and to try and avoid the secondary peak in oestrogen levels (Legro et al. 2008), OCP user's oestrogen levels were tested on the 7th pill taking day (Elliott et al. 2005). The 7th pill taking day is equivalent to the 14th day of the overall pill cycle ( 7 pill-free days plus 7 pill taking days), therefore the measurement day will be referred to as the 14th day of the cycle from this point forward. To avoid an acute peak in oestrogen levels the OCP users were not tested within $2 \mathrm{~h}$ of taking their OCP (Sneader 2005). The design and several of the measurement techniques within the current study have been reported previously (Hicks et al. 2013). The testing sessions were as follows: (1) pre-damage; (2) damage (48 h post pre-damage); (3) $48 \mathrm{~h}$; (4) $96 \mathrm{~h}$ and (5) $168 \mathrm{~h}$ post-damage (see Fig. 1). Pre-damage assessments consisted of stature and mass (anthropometric measures), patella tendon moment arm, 5-6 ml blood sample (for serum CK levels), dynamometer familiarisation (within pre-damage), morphological and mechanical measures of the patella tendon (tendon size and stiffness, respectively) and maximal voluntary isometric knee extension $\left(\mathrm{MVC}_{\mathrm{KE}}\right)$ torque measurements at six angles $\left[60^{\circ}, 65^{\circ}, 70^{\circ}, 75^{\circ}, 80^{\circ}\right.$ and $90^{\circ}$ (full extension $=0^{\circ}$ ) $]$. Participants performed two practice maximal voluntary contractions, at two knee joint angles during the familiarisation session. Stature and mass were measured using a wall-mounted stadiometer (Harpenden, Holtain Crymych, UK) and digital scales (Seca model 873, Seca, Germany), respectively. The damage session was on the 14th day of the cycle (pill or menstrual) in OCP users and non-users. The damage session consisted of maximal voluntary eccentric knee contractions $\left(\mathrm{MVE}_{\mathrm{KE}}\right), 5-6 \mathrm{ml}$ venous blood sample (for serum $\mathrm{CK}$ and oestrogen levels), rating of muscle soreness and $\mathrm{MVC}_{\mathrm{KE}}$ torque measurements. 48, 96 and $168 \mathrm{~h}$ testing session consisted of 5-6 $\mathrm{ml}$ blood sample (for serum CK levels), rating of muscle soreness, and $\mathrm{MVC}_{\mathrm{KE}}$ torque measurements at six angles $\left(60^{\circ}, 65^{\circ}, 70^{\circ}, 75^{\circ}, 80^{\circ}\right.$ and $\left.90^{\circ}\right)$.

All tests were carried out in the non-dominant leg. The non-dominant leg was defined as the leg that provided stability during movements such as kicking a ball. Participants were seated in an isokinetic dynamometer (Cybex Norm, Cybex International, NY, USA), with a hip angle of $85^{\circ}$. To reduce any extraneous movement during maximal efforts, participants were secured in a seated position using inextensible straps secured around the shoulders and hips. The isokinetic dynamometer axis of rotation was visually aligned with the knee joint's centre of rotation. During pre-damage, the isokinetic dynamometer settings (chair position, distance from the dynamometer, dynamometer arm length) and anatomical zero (leg fully extended, knee angle $0^{\circ}$ ) were recorded to ensure accurate repeatability in the following sessions.

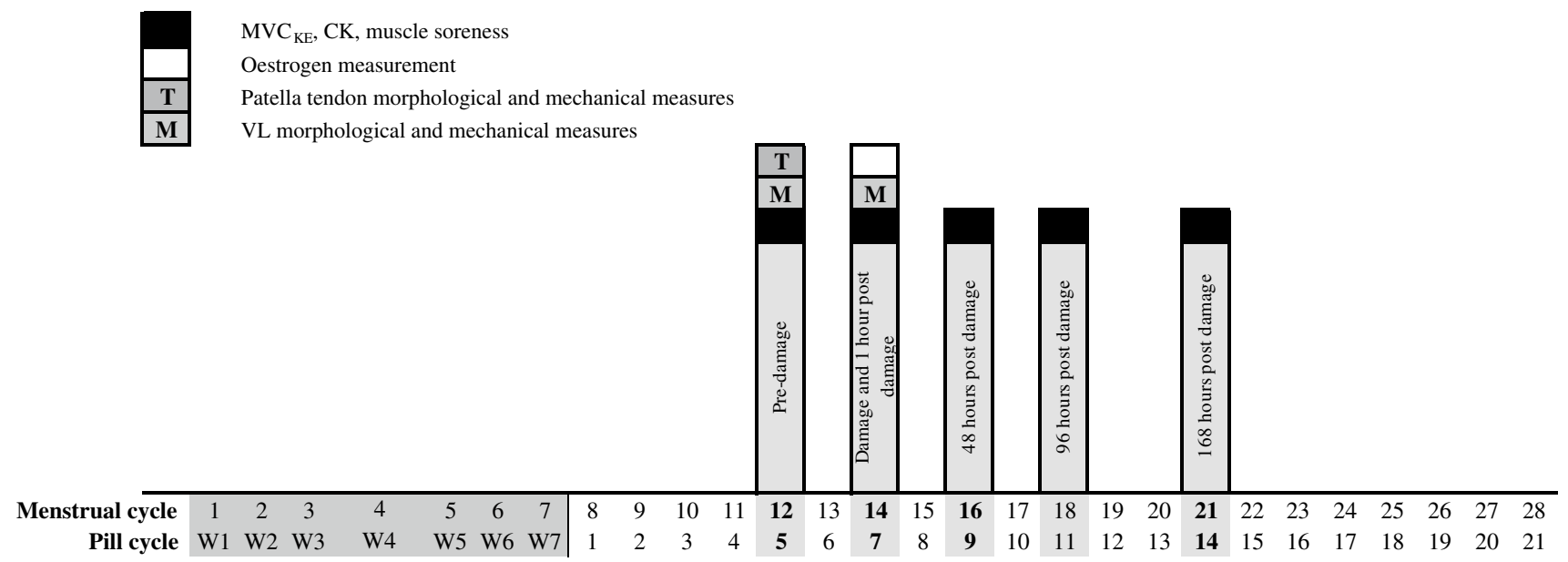

Fig. 1 Schematic diagram to illustrate the testing timeline throughout the cycle (pill or menstrual) in OCP users and non-users. $M V C_{K E}$ maximal voluntary isometric knee extension, $C K$ creatine kinase, $V L$ vastus lateralis 


\section{Vastus lateralis anatomical cross-sectional area}

The method used to determine vastus lateralis (VL) crosssectional area has been reported in detail previously (Hicks et al. 2013). In brief, in a supine position, VL anatomical cross-sectional area $\left(\mathrm{VL}_{\mathrm{ACSA}}\right)$ was measured using a realtime B-mode ultrasound (AU5 Harmonic, Esaote Biomedica, Genoa, Italy). At $50 \%$ of VL muscle length echoabsorptive markers (Transpore surgical tape, Medisave UK Ltd, UK), were placed in parallel at intervals of $30 \mathrm{~mm}$. The ultrasound probe $(7.5 \mathrm{MHz}$ linear array probe, $38 \mathrm{~mm}$ wide), was held perpendicular to the VL muscle in the axial plane, and moved steadily over the echo-absorptive markers from the lateral to the medial edge of the muscle. The images were recorded in real-time at 25 frames per second (Adobe Premier pro Version 6, Adobe Systems Software, Ireland). Using video editing software (Adobe Premier Elements, version 10), still images were acquired at each $30 \mathrm{~mm}$ interval. The shadows cast by the echo-absorptive markers allowed the neighbouring still images to be aligned, thus reconstructing the entire $\mathrm{VL}_{\mathrm{ACSA}}$ in a single image (Adobe Photoshop Elements, version 10). Digitising software (ImageJ 1.45, National Institutes of Health, USA) was used to measure $\mathrm{VL}_{\mathrm{ACSA}}$.

\section{Torque measurements}

At six different knee angles $\left[60^{\circ}, 65^{\circ}, 70^{\circ}, 75^{\circ}, 80^{\circ}\right.$ and $90^{\circ}$ (full extension $=0^{\circ}$ )], participants were instructed to perform two $\mathrm{MVC}_{\mathrm{KE}}$ lasting $\sim 2 \mathrm{~s}$ at the plateau, with $90 \mathrm{~s}$ rest between contractions. Torque was presented, in realtime, on a Macintosh G4 computer (Apple Inc., Cupertino, CA, USA), via an A/D converter (Biopac Systems, Santa Barbara, CA, USA). Torque measurements were later analysed offline with the accompanying software (Acknowledge, version 3.9.2). The highest peak torque produced at each angle was taken as $\mathrm{MVC}_{\mathrm{KE}}$ peak torque. The knee angle where the highest torque was produced was recorded as optimal knee angle. In a randomised order, $\mathrm{MVC}_{\mathrm{KE}}$ torque measurements were repeated at all six knee angles $60 \mathrm{~min}$ post-eccentric exercise [to reduce any fatigue effect (Walsh et al. 2004)] and 48, 96 and $168 \mathrm{~h}$ post-eccentric exercise. $\mathrm{MVC}_{\mathrm{KE}}$ torque loss was calculated from optimal knee angle identified at pre-damage.

\section{Patella tendon length and cross-sectional area}

A real-time B-mode ultrasound (AU5 Harmonic, Esaote Biomedica, Genoa, Italy) was used to measure patella tendon cross-sectional area and patella tendon length at a fixed $90^{\circ}$ knee angle. The distance between the apex of the patella and the tibial tuberosity, marked using sagittal ultrasound images, was taken as patella tendon length. To measure patella tendon cross-sectional area, the ultrasound probe was placed in the transverse plane and images were captured at 25,50 , and $75 \%$ of patella tendon length. The images were later analysed offline using image analyses software, Image (1.45, National Institutes of Health, USA) patella tendon cross-sectional area is presented as a mean of all three images (O’Brien et al. 2010).

\section{Patella tendon stiffness}

The participants were seated in the isokinetic dynamometer, with the knee angle fixed at $90^{\circ}$, and were instructed to perform a ramped, isometric $\mathrm{MVC}_{\mathrm{KE}}$ lasting 5-6 s. Ramped $\mathrm{MVC}_{\mathrm{KE}}$ torque and displacement of the patella tendon were synchronised using a $10-\mathrm{V}$ square wave, signal generator. Patella tendon displacement was measured over two $\mathrm{MVC}_{\mathrm{KE}}$, once with the ultrasound probe positioned over the distal edge of the patella and on the second contraction over the tibial tuberosity (Onambélé et al. 2007), so that total displacement would be computed from the composite of proximal and distal patella motions (see below). Torque was presented on a Macintosh G4 computer (Apple Inc., Cupertino, CA, USA), via an A/D converter and subsequently analysed with the accompanying software (Acknowledge, Biopac Systems, Santa Barbara, CA, USA). To create an external marker on the ultrasound images, an echo-absorptive marker was placed on the skin. Using the marker to calculate displacement, the distance of the marker (shadow) from an anatomical reference point at the beginning of the contraction to the position of the shadow at the end of the contraction was calculated. A square wave, signal generator was used to synchronise the ultrasound images with the torque acquisition system. Images were captured at $\sim 10 \%$ intervals of ramped $\mathrm{MVC}_{\mathrm{KE}}$ torque (Onambélé et al. 2007). Total patella tendon displacement was calculated as displacement at the apex of the patella plus the displacement at the tibial tuberosity (Onambélé et al. 2007). Patella tendon forces were calculated as: $\left(\mathrm{MVC}_{\mathrm{KE}}\right.$ torque + antagonist co-activation torque)/patella tendon moment arm.

Patella tendon moment arm was measured at $90^{\circ}$ (full extension $=0^{\circ}$ ) in the sagittal plane, from a dual-energy X-ray absorptiometry scan (frame $23.3 \mathrm{~cm} \times 13.7 \mathrm{~cm}$, Hologic Discovery, Vertec Scientific Ltd, UK), and subsequently analysed using a DICOM image assessment tool (OsiriX DICOM viewer, ver. 4.0, Pixemo, Switzerland). Patella tendon moment arm length was determined as the perpendicular distance from the centre of the patella tendon to the tibio-femoral contact point. Dual-energy X-ray absorptiometry scans have recently been shown to be a reliable and valid method of measuring patella tendon moment arm length when compared to the gold standard method (MRI) (Erskine et al. 2014). 
For the calculation of tendon force, the calculation of antagonist co-activation torque is described below. The patella tendon force-elongation curve constructed from data analysed at every $10 \% \mathrm{MVC}_{\mathrm{KE}}$, was then fitted with a second-order polynomial function forced through zero (Onambélé et al. 2007). The tangential slope at discreet sections of the curve, relative to $\mathrm{MVC}_{\mathrm{KE}}$ force, was calculated by differentiating the curve at every $10 \%$ patella tendon force interval. In addition, to standardise the comparison of patella tendon stiffness at an absolute load, the slope of the tangential line, corresponding to the $\mathrm{MVC}_{\mathrm{KE}}$ force of the weakest participant, was computed for each subject.

\section{Co-activation during maximal voluntary isometric knee extension}

In order to compute patella tendon force, the co-activation of the biceps femoris during $\mathrm{MVC}_{\mathrm{KE}}$ was measured. To determine co-activation of the biceps femoris during the ramped $\mathrm{MVC}_{\mathrm{KE}}$, electrodes (Ambu, Neuroline 720, Denmark) were placed in a bipolar configuration at $25 \%$ of biceps femoris muscle length (distal end $=0 \%$ ). A reference electrode (Ambu, Blue Sensor, Denmark) was placed on the lateral tibial condyle. The raw electromyography signal was amplified $(\times 2000)$ and filtered (through low and high pass filters of 10 and $500 \mathrm{~Hz}$, respectively, notch at $50 \mathrm{~Hz}$ ) with the sampling frequency set at $2000 \mathrm{~Hz}$. Ramped $\mathrm{MVC}_{\mathrm{KE}}$ torque and biceps femoris electromyography were recorded in real-time and synchronised using a $(10-\mathrm{V})$ square wave signal generator. Participants performed two maximal voluntary isometric knee flexions $\left(\mathrm{MVC}_{\mathrm{KF}}\right)$ at $90^{\circ}$. They were instructed to perform $\mathrm{MVC}_{\mathrm{KF}}$ rapidly and as forcefully as possible and instructed to relax once a 2-s plateau had been attained (as observed on the dynamometer screen display). The root mean square of the biceps femoris electromyography signal was calculated $500 \mathrm{~ms}$ either side of instantaneous $\mathrm{MVC}_{\mathrm{KF}}$ maximal torque from the contraction corresponding to the highest $\mathrm{MVC}_{\mathrm{KF}}$ torque. Prior to contraction the baseline signal noise was calculated as the integral root mean square over $1 \mathrm{~s}$ and removed from the measured electromyography during $\mathrm{MVC}_{\mathrm{KF}}$ and $\mathrm{MVC}_{\mathrm{KE}}$. At every $10 \%$ of ramped $\mathrm{MVC}_{\mathrm{KE}}$ torque the absolute integral of the biceps femoris electromyography was taken over $250 \mathrm{~ms}$. Co-activation torque was calculated as: (biceps femoris electromyography during ramped $\mathrm{MVC}_{\mathrm{KE}}$ /biceps femoris electromyography during $\left.\mathrm{MVC}_{\mathrm{KF}}\right) \times \mathrm{MVC}_{\mathrm{KF}}$ torque at $90^{\circ}$ knee angle (Onambélé et al. 2007). This equation assumes that the biceps femoris is representative of the entire hamstring (Carolan and Cafarelli 1992) and that a linear relationship exists between biceps femoris electromyography and $\mathrm{MVC}_{\mathrm{KF}}$ torque (Lippold 1952).

\section{Patella tendon stress/strain relationship}

Patella tendon strain was calculated as a ratio of total patella tendon displacement and patella tendon length. Patella tendon stress was calculated as: patella tendon force $(\mathrm{N}) /$ patella tendon cross-sectional area $\left(\mathrm{mm}^{2}\right)$.

\section{Young's modulus}

Young's modulus was calculated as: patella tendon stiffness $\times$ [patella tendon length $(\mathrm{mm}) /$ patella tendon crosssectional area $\left.\left(\mathrm{mm}^{2}\right)\right]$.

\section{'Damaging' eccentric exercise}

The damaging protocol used within the study has been described in detail previously (Hicks et al. 2013). Following a warm-up of 10 isokinetic knee extensions and knee flexions, participants performed six sets of $12 \mathrm{MVE}_{\mathrm{KE}}$. For the eccentric exercise, the knee extension range of motion was set at $20^{\circ}-90^{\circ}\left(0^{\circ}=\right.$ full extension $)$. The eccentric phase of the contractions was performed at an isokinetic angular velocity of $30 \%$ s (Jamurtas et al. 2005). The concentric phase was performed passively at an angular velocity of $60 \%$ s. Two minutes rest was provided between each set. Participants remained seated in the isokinetic dynamometer throughout the entire exercise protocol, including rest periods. Visual feedback and verbal encouragement were continuously provided throughout the protocol. $\mathrm{MVE}_{\mathrm{KE}}$ torque was recorded throughout each contraction and displayed via the torque acquisition system. For each set, peak $\mathrm{MVE}_{\mathrm{KE}}$ torque was determined as the highest torque out of the 12 repetitions. Average peak $\mathrm{MVE}_{\mathrm{KE}}$ torque was calculated as an average of peak $\mathrm{MVE}_{\mathrm{KE}}$ across six sets.

\section{Muscle soreness}

Muscle soreness was measured using a visual analogue scale. The visual analogue scale consisted of a $100 \mathrm{~mm}$ line, with $0 \mathrm{~mm}$ labelled "No pain at all" and $100 \mathrm{~mm}$ labelled "Unbearable pain". Seated in the isokinetic dynamometer, the leg was passively moved through a full range of motion at $30 \%$ s. Participants were asked to mark a line perpendicular to the visual analogue scale to denote the level of pain they experienced during the passive movement. The visual analogue scale has been reported to be a reliable measure of muscle soreness [ICC > 0.96 (Bijur et al. 2001)].

\section{Blood samples}

Venous blood samples were taken to measure CK and oestradiol levels. A 21-gauge needle was inserted into the antecubital vein of the forearm using a $10 \mathrm{ml}$ syringe. 
5-6 $\mathrm{ml}$ of blood was drawn into a serum collection tube. The sample was left on a crushed ice bed for $60 \mathrm{~min}$. The sample was then centrifuged at $4500 \mathrm{rpm}$ at $0{ }^{\circ} \mathrm{C}$ for $10 \mathrm{~min}$. Using a 200-1000 $\mu \mathrm{l}$ pipette (Eppendorf, Hamburg, Germany), the resulting serum sample was separated into three aliquots $(\sim 500 \mu \mathrm{l}$ each) and stored in eppendorfs at $-20{ }^{\circ} \mathrm{C}$ until $\mathrm{CK}$ and oestradiol analysis was performed.

Creatine kinase levels were measured using a standard colorimetry procedure, measuring at optical density $340 \mathrm{~nm}$ (BioTek ELx800 96 well Microplate Reader) and immediately analysed (Gen5, version 2.0). Each sample was run in duplicate-quadruplets using an EnzyChrom ${ }^{\mathrm{TM}} \mathrm{CK}$ Assay Kit (BioAssay Systems, Hayward, CA, USA, sensitivity $5 \mathrm{U} / \mathrm{l}$, manufacturer intra-assay variability $\leq 5 \%$ ). An average of 2-4 readings were taken. Creatine kinase activity is reported in two ways, firstly as absolute values and secondly, as the increase from pre to peak CK over the $168 \mathrm{~h}$ $\left[\left(\Delta \mathrm{CK}_{\text {peak }}\right)\right.$, i.e. the peak $\mathrm{CK}$ value - the pre-CK values].

Oestradiol was measured using a standard enzymelinked immunosorbent assay (ELISA) procedure (Alpha Diagnostic International, San Antonio, USA). Absorbance was measured at optical density of $450 \mathrm{~nm}$ (BioTek ELx800 96 well Microplate Reader). The minimal oestradiol detection was $\sim 10 \mathrm{pg} / \mathrm{ml}$, the manufacturers intra-precision and inter-precision was 9.85 and $10.3 \%$, respectively. To calculate oestrogen levels, a standard curve was plotted using the six standards against their absorbance. Using the mean absorbance of each sample, the concentration of the sample was read directly from the standard curve.

\section{Statistics}

Statistical analyses were carried out using the statistical software package SPSS (v.19, Chicago, IL, USA) for Windows and Microsoft Excel. To ensure that the data were parametric, the Levene's and Shapiro-Wilk's tests were used to assess the variance and normality of the data. If parametric tests were violated, the equivalent non-parametric tests were used. For group differences in anthropometric measures, oestrogen levels, $\mathrm{VL}_{\mathrm{ACSA}}$, patella tendon properties, and $\Delta \mathrm{CK}_{\text {peak }}$ independent $T$ tests were used. A $2 \times 5$ mixed design analysis of variance [ANOVA, between factors: OCP use (2 levels) and time from EIMD (5 levels)] was used for muscle soreness, $\mathrm{MVC}_{\mathrm{KE}}$ torque loss and $\mathrm{CK}$ levels. Wherever the assumption of sphericity was violated, the Greenhouse-Geisser correction was used. When a significant group effect was found a planned contrast, with LSD correction, was used to identify where the significant difference lay. Since an association was reported between age and $\mathrm{MVC}_{\mathrm{KE}}$ torque loss, an analysis of covariance was used. For the variables being investigated as a potential determinant of EIMD, confidence levels and Cohen $d$ were calculated. Significance was set at $p \leq 0.05$. The data are presented as mean \pm standard deviation.

\section{Results}

\section{Anthropometric measurements}

OCP users were significantly older by 2.2 years $(p=0.010)$ and of an $11 \%$ greater mass $(p=0.047)$ than non-users. There was no significant difference in stature between OCP users and non-users $(p=0.061)$.

\section{Oestrogen levels}

Serum oestrogen levels were significantly lower in OCP users compared to non-users on the 14th day of the cycle (pill or menstrual) $(209 \pm 115$ and $433 \pm 147 \mathrm{pg} / \mathrm{ml}$, respectively, $p=0.004$ ).

\section{$\mathbf{V L}_{\mathrm{ACSA}}$}

There was no significant difference in $\mathrm{VL}_{\mathrm{ACSA}}$ between OCP users and non-users $(21.9 \pm 2.64$ and $20.1 \pm 3.40 \mathrm{~cm}^{2}$, respectively, $p=0.224$ ).

\section{Tendon properties}

Patella tendon properties for OCP users and non-users are presented in Table 1. Patella tendon length, patella tendon cross-sectional area and patella tendon moment arm were not significantly different between OCP users and nonusers $(p \geq 0.05)$. Furthermore, ramped $\mathrm{MVC}_{\mathrm{KE}}$ torque and patella tendon force were not significantly different between OCP users and non-users $(p \geq 0.05)$. Patella tendon elongation was not significantly different between OCP users and non-users $(7.20 \pm 1.30,8.16 \pm 0.80 \mathrm{~mm}$, respectively, $p=0.075$ ). Tendon stiffness (Fig. 2) at $\mathrm{MVC}_{\mathrm{KE}}$ was not

Table 1 Patella tendon properties in OCP users and non-users

\begin{tabular}{|c|c|c|}
\hline & OCP users & Non-users \\
\hline Patella tendon length (mm) & $52.9 \pm 4.3$ & $49.0 \pm 6.0$ \\
\hline $\begin{array}{l}\text { Mean patella tendon cross-sectional area } \\
\left(\mathrm{mm}^{2}\right)\end{array}$ & $63.2 \pm 15.1$ & $56.0 \pm 17.2$ \\
\hline Patella tendon moment arm $(\mathrm{cm})$ & $4.01 \pm 0.24$ & $3.96 \pm 0.28$ \\
\hline Maximal ramped $\mathrm{MVC}_{\mathrm{KE}}$ torque $(\mathrm{Nm})$ & $143 \pm 30$ & $132 \pm 27$ \\
\hline Maximal patella tendon force $(\mathrm{N})$ & $3634 \pm 717$ & $3430 \pm 768$ \\
\hline Maximal patella tendon stiffness $(\mathrm{N} / \mathrm{mm})$ & $872 \pm 366$ & $665 \pm 169$ \\
\hline Maximal Young's modulus (MPa) & $761 \pm 331$ & $642 \pm 285$ \\
\hline
\end{tabular}

Patella tendon stiffness and Young's modulus are presented at $100 \%$ ramped maximal voluntary isometric knee extension. Patella tendon moment arm was measured at $90^{\circ}$ knee angle 
significantly different between OCP users and non-users $[p=0.153$, CI $(-502,88.0), d=0.73]$. Young's modulus was not significantly different between OCP users and nonusers [ $p=0.213, \mathrm{CI}(-190,428), d=0.38]$. Patella tendon stiffness calculated at a standardised patella tendon force $(2330 \mathrm{~N})$ was not significantly different between OCP users and non-users $(725 \pm 219$ and $565 \pm 103 \mathrm{~N} / \mathrm{mm}$, respectively $[p=0.070, \mathrm{CI}(-10.9,331), d=0.94]$. Patella tendon Young's modulus calculated at a standardised patella tendon force $(2330 \mathrm{~N})$ was not significantly different between OCP users and non-users $[628 \pm 219$ and $536 \pm 190 \mathrm{MPa}$, respectively $(p=0.175 \mathrm{CI}(-111,298), d=0.453)]$.

\section{Torque production during $\mathrm{MVE}_{\mathrm{KE}}$}

There was no significant difference in the average peak $\mathrm{MVE}_{\mathrm{KE}}$ torque of each set (six in total) between OCP users and non-users $(p \leq 0.05)$. There was no significant difference in the average peak $\mathrm{MVE}_{\mathrm{KE}}$ torque (averaged over 6 sets) between OCP users and non-users (182 \pm 33 and $167 \pm 32 \mathrm{Nm}$, respectively, $p=0.093$ ). Peak $\mathrm{MVE}_{\mathrm{KE}}$ torque made relative to pre- $\mathrm{MVC}_{\mathrm{KE}}$ torque was significantly higher in OCP users compared to non-users $(108 \pm 16.7$ and $85.0 \pm 11.9 \%$, respectively, $p=0.002)$.

\section{Change in optimal knee angle}

Pre-damage, optimal $\mathrm{MVC}_{\mathrm{KE}}$ knee angle was not significantly different between OCP users and non-users (median, $75.0 \pm 7.0^{\circ}$, and $75.0 \pm 7.0^{\circ}$, respectively, $p=0.430$ ). Post-EIMD both OCP users $\left(80.0 \pm 7.0^{\circ}, p=0.010\right)$ and non-users (median $80.0 \pm 7.0^{\circ}, p=0.030$ ) demonstrated a significant rightward shift in $\mathrm{MVC}_{\mathrm{KE}}$ optimal angle. There was no significant difference in the magnitude of the rightward shift in $\mathrm{MVC}_{\mathrm{KE}}$ optimal angle between $\mathrm{OCP}$ users and non-users $(p=0.311)$.

\section{$\mathrm{MVC}_{\mathrm{KE}}$ torque loss}

$\mathrm{MVC}_{\mathrm{KE}}$ torque loss expressed as a percentage of predamage $\mathrm{MVC}_{\mathrm{KE}}$ torque is illustrated in Fig. 3. A twoway repeated mixed ANOVA for $\mathrm{MVC}_{\mathrm{KE}}$ torque loss post-EIMD, reported a significant main effect of time ( $p=0.0004)$; however, no group effect (OCP users versus non-users $p=0.257$ ) nor interaction (times versus group, $p=0.118$ ) was reported. Pre-MVC $\mathrm{KE}_{\mathrm{KE}}$ peak torque was not significantly different between OCP users and non-users $(169 \pm 24.3$ and $194 \pm 36.2 \mathrm{Nm}, p=0.055)$. Although a significant tendency was reported, peak $\mathrm{MVC}_{\mathrm{KE}}$ torque loss made relative to pre-damage $\mathrm{MVC}_{\mathrm{KE}}$ torque was not significantly different between OCP users compared to non-users $(17 \pm 7$ and $28 \pm 13 \%$, respectively $[p=0.055, \mathrm{CI}(-21.5$, $10.5), d=0.94)]$.

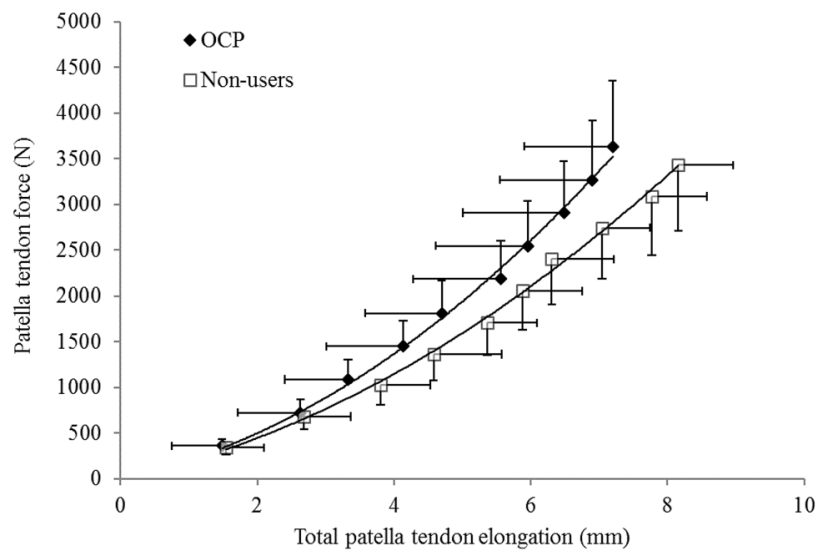

Fig. 2 Patella tendon force-elongation relationship, during ramped maximal voluntary isometric contraction, in oral contraceptive pill user and non-users. Data are presented as mean \pm standard deviation

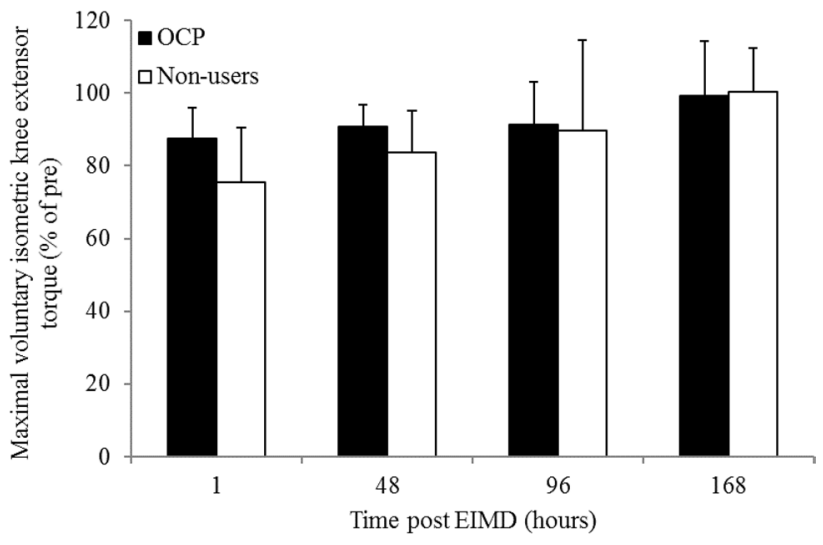

Fig. 3 Maximal voluntary isometric knee extensor torque in oral contraceptive pill users and non-users expressed as a percentage of maximal voluntary isometric knee extensor torque pre-damage. Data are presented as mean \pm standard deviation

A significant correlation was observed between age and $\mathrm{MVC}_{\mathrm{KE}}$ torque loss $(r=0.58, p=0.011)$. Age was therefore considered as a covariate of $\mathrm{MVC}_{\mathrm{KE}}$ torque loss. A subsequent ANCOVA revealed that no significant difference in $\mathrm{MVC}_{\mathrm{KE}}$ torque loss between OCP users and nonusers remained when age was accounted for $(p=0.179)$.

\section{Creatine kinase levels}

A two-way repeated mixed ANOVA for CK reported a significant main effect of time $(p=0.040)$; however, no significant group (OCP users versus non-users, $p=0.057$ ) or interaction (time versus group, $p=0.576$ ) effects were reported (Fig. 4). $\triangle \mathrm{CK}_{\text {peak }}$ was significantly higher in OCP users compared to non-users over the $168 \mathrm{~h}[962 \pm 968$ and 


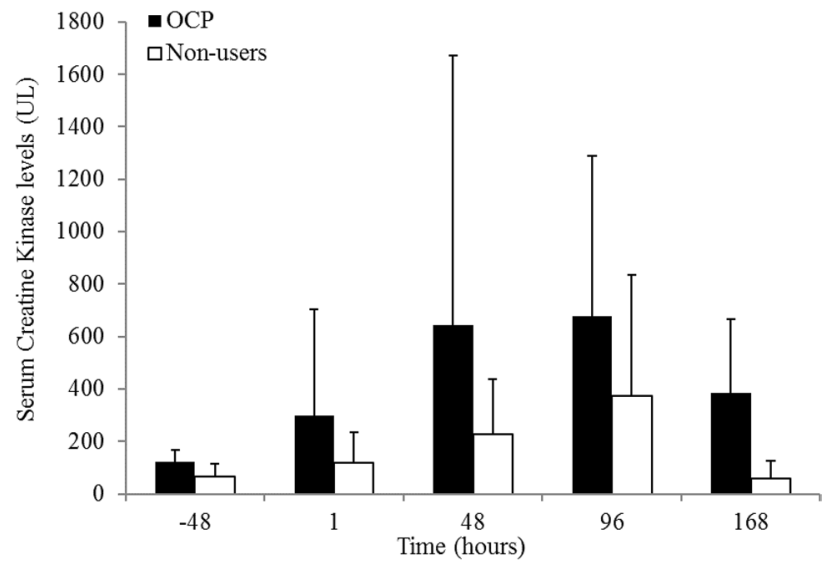

Fig. 4 Creatine kinase response in oral contraceptive pill users and non-users pre $(-48 \mathrm{~h})$ and post-exercise induced muscle damage. Data are presented as mean \pm standard deviation

$386 \pm 474$ UL, respectively $(p=0.016$, CI $(-186,1338)$, $d=0.76)]$.

\section{Muscle soreness}

A two-way mixed measures ANOVA for muscle soreness reported a significant main effect of time $(p=0.0004)$; however, there was no significant group difference (OCP users versus non-users, $p=0.598$ ) or an interaction effect $(p=0.127)$. For both OCP users and non-users peak muscle soreness occurred $48 \mathrm{~h}$ post-EIMD. There was no significant difference in peak muscle soreness between OCP users and non-users ( $40 \pm 3 \mathrm{~mm}$ and $41 \pm 1 \mathrm{~mm}$, respectively, $p=0.358$ ).

\section{Discussion}

The current study found that (1) the CK response was augmented in OCP users compared to non-users; however, $\mathrm{MVC}_{\mathrm{KE}}$ torque loss and muscle soreness were not different; (2) the patella tendon properties were not significantly different between the OCP users and non-users and, as such, did not contribute to the observed significant difference in EIMD.

Within the current study, the circulating oestrogen levels within the OCP users were 2.8 times higher than oestrogen levels within OCP users reported by Bryant et al. (2008). This discrepancy in oestrogen levels may be confounded by a secondary rise in oestrogen levels following the pillfree interval period within the current study. Despite elevated levels within OCP users, in agreement with previous research (Bryant et al. 2008), the current study confirms a significant down regulation of oestrogen within OCP users compared to non-users. Thus it is reasonable to assume that in the present study, the down regulation of oestrogen in the OCP users compared to the non-users and the suppression of oestrogen in the OCP users, remained throughout the cycle (Bryant et al. 2008).

Due to high levels of oestrogen attenuating circulating levels of IGF-I (Heald et al. 2005) it can be expected that the anabolic effect of IGF-I on tendon fibroblast and therefore tendon collagen synthesis is greater in OCP users compared to non-users. Subsequently, higher tendon stiffness in OCP users compared to non-user may be expected. Within the current study, however, despite significantly lower oestrogen levels in OCP users, there was no significant difference in patella tendon stiffness in vivo between OCP users and non-users. Although our data show a trend towards higher stiffness in the OCP users, our findings did not reach significance and contradict those of Bryant et al. (2008), but are in agreement with those of Hansen et al. (2013). Bryant et al. (2008) concluded that exposure to the OCP ( $\geq 1$ year) decreases Achilles tendon strain properties. These discrepancies may be attributed to the fact that Bryant et al. (2008) reported data from the Achilles tendon, whereas the present study assessed the patella tendon. Therefore, it is possible that the Achilles and patella tendons show inconsistent responses to the OCP due to the difference in the number of oestrogen receptor sites, as our findings concur with those of Hansen et al. (2013). Hansen et al. (2013) reported no significant difference in patella tendon stiffness or Young's modulus between OCP users and non-users. The current study confirms that the OCP does not alter patella tendon stiffness or Young's modulus in recreationally active participants. The conclusion that the OCP has no significant effect on tendon properties, specifically patella tendon stiffness, may discount any potential differences in EIMD between OCP users and non-users being directly attributed to patella tendon properties.

As a marker of muscle damage, the current study reported no significant difference in $\mathrm{MVC}_{\mathrm{KE}}$ torque loss between OCP users and non-users, which both supports (Sewright et al. 2008) and contradicts (Minahan et al. 2015; Savage and Clarkson 2002) previous research. Within the knee extensors, our findings directly contradict those of Minahan et al. (2015) who reported torque loss $48 \mathrm{~h}$ postEIMD to be significantly higher in OCP users compared to non-users. The discrepancies may be attributed to Minahan et al. (2015) reporting substantially lower levels of oestrogen in the OCP users compared to the current study. Differing levels of oestrogen may be explained by Minahan et al. (2015) measuring oestrogen during the 7 days pill-free period, whereas the current study measured oestrogen on the 14th day (7th pill day) and the mix of OCP brands used within both studies (Elliott-Sale et al. 2013). Although the current study tested on the 7th day of the pill cycle to try and avoid the secondary peak in oestrogen, testing on the 
10th day of the pill cycle (Legro et al. 2008) and controlling the type of OCP used (Elliott-Sale et al. 2013) may be required to ensure OCP oestrogen levels are plateaued and comparable, respectively. Therefore, although a significant difference in oestrogen levels between OCP users and nonusers was reported within the current study, the augmented oestrogen levels within the OCP users when measured on day 7 of the pill cycle may have masked the effect of OCP use on torque loss post-EIMD. Therefore, the current study concludes that on the 14th day of the cycle (pill or menstrual), there is no significant difference in $\mathrm{MVC}_{\mathrm{KE}}$ torque loss between OCP users and non-users post-EIMD.

Although torque loss is regarded as the most functional measure of EIMD (Warren et al. 1999), in agreement with previous research (Joyce et al. 2014; Minahan et al. 2015; Roth et al. 2001), the current study reported $\Delta \mathrm{CK}_{\text {peak }}$ to be significantly higher in OCP users (low oestrogen levels) compared to non-users (high oestrogen levels) following EIMD. Although Joyce et al. (2014) and Minahan et al. (2015) reported CK levels to be significantly higher in OCP users compared to non-users, they only monitored CK up to $48 \mathrm{~h}$ post-EIMD despite peak CK characteristically occurring at $96 \mathrm{~h}$ post-EIMD (Hyatt and Clarkson 1998). By measuring CK at 96 and $168 \mathrm{~h}$ post-EIMD, the current study reduces the chance of missing a true peak in CK and can therefore confirm that $\Delta \mathrm{CK}_{\text {peak }}$ is significantly higher in OCP users compared to non-users post-EIMD. In apparent contrast to our findings, $72 \mathrm{~h}$ post-EIMD, Carter et al. (2001) observed OCP users to have significantly lower CK levels compared to non-users. It should be noted, however, that we carried out EIMD on day 14 of the menstrual cycle, whereas Carter et al. (2001) carried out EIMD on day 22 (midluteal phase). In the present study, we observed oestrogen levels to be significantly lower in OCP users compared to non-users, whereas Carter et al. (2001) showed OCP users to higher oestrogen levels compared to non-users. Ignoring the OCP status of Carter et al. (2001) participants, at the time of testing, the group with the lowest oestrogen level showed significantly higher CK levels post-EIMD. This is therefore consistent with our findings, that the lower oestrogen levels (observed within our OCP users) results in a higher $\Delta \mathrm{CK}_{\text {peak }}$ post-EIMD.

Oestrogen has been reported to decrease cell membrane fluidity, thus increase cell membrane stability. An increase in cell membrane stability may prevent muscle proteins (i.e. CK) leaking into the interstitial space and into the systemic circulation (Carter et al. 2001; Wiseman et al. 1993) and attenuate the influx of neutrophils and cytokines post-EIMD (MacNeil et al. 2011; Silvestri et al. 2003). In support of oestrogen preventing the leakage of CK from the intracellular membrane, Carter et al. (2001) reported a moderate correlation $(r=-0.43, p<0.05)$ between total resting oestrogen levels and overall CK. Therefore, the potential effects of oestrogen on cell membrane stability may explain the differences in CK levels between OCP users and non-users within the current study; however, muscle biopsies would be required to confirm such conclusions.

Although the current study controlled for the type (monophasic) and the dosage of synthetic oestrogen level within the OCP, it must be noted that the oestrogen levels vary between different brands of OCP (Elliott et al. 2005). Therefore, to fully understand the effect of OCP on EIMD, a specific brand needs to be investigated. Furthermore, due to the natural fluctuation of oestrogen levels during the menstrual cycle and the repeated measure design of the current study, measurement of oestrogen levels at every time point, rather than just on day 14 of the menstrual cycle, may be required in future studies.

As no other significant differences between suggested determinants of EIMD in OCP users and non-users (tendon properties and $\mathrm{MVE}_{\mathrm{KE}}$ torque) have been reported, the current study concludes that the higher $\triangle \mathrm{CK}_{\text {peak }}$ in OCP users may be attributed to the significantly higher oestrogen levels in non-users. Therefore, the current study concludes that OCP use does not increase susceptibility to functional impairment $\left(\mathrm{MVC}_{\mathrm{KE}}\right.$ torque loss) post-EIMD, but it does augment the $\mathrm{CK}$ response post-EIMD.

Acknowledgements The study was funded and supported by the Institute for Performance Research at Manchester Metropolitan University Cheshire.

\section{Compliance with ethical standards}

Conflict of interest The authors declare that no conflict of interest exists.

Open Access This article is distributed under the terms of the Creative Commons Attribution 4.0 International License (http://creativecommons.org/licenses/by/4.0/), which permits unrestricted use, distribution, and reproduction in any medium, provided you give appropriate credit to the original author(s) and the source, provide a link to the Creative Commons license, and indicate if changes were made.

\section{References}

Amelink G, Koot R, Erich W, Gijn J, Bär P (1990) Sex-linked variation in creatine kinase release, and its dependence on oestradiol, can be demonstrated in an in vitro rat skeletal muscle preparation. Acta Physiol Scand 138:115-124

Amelink G, Van der Wal W, Wokke J, Van Asbeck B, Bär P (1991) Exercise-induced muscle damage in the rat: the effect of vitamin E deficiency. Pflüg Arch 419:304-309

Bär P, Amelink G, Oldenburg B, Blankenstein M (1988) Prevention of exercise-induced muscle membrane damage by oestradiol. Life Sci 42:2677-2681 
Bijur PE, Silver W, Gallagher EJ (2001) Reliability of the visual analog scale for measurement of acute pain. Acad Emerg Med 8:1153-1157

Brown J (1955) Urinary excretion of oestrogens during the menstrual cycle. Lancet 265:320-323

Bryant AL et al (2008) Effects of estrogen on the mechanical behavior of the human Achilles tendon in vivo. J Appl Physiol 105:1035-1043

Carolan B, Cafarelli E (1992) Adaptations in coactivation after isometric resistance training. J Appl Physiol 73:911

Carter A, Dobridge J, Hackney A (2001) Influence of estrogen on markers of muscle tissue damage following eccentric exercise. Hum Physiol 27:626-630

Chen TC, Lin K-Y, Chen H-L, Lin M-J, Nosaka K (2011) Comparison in eccentric exercise-induced muscle damage among four limb muscles. Eur J Appl Physiol 111:211-223

Cole LA, Ladner DG, Byrn FW (2009) The normal variabilities of the menstrual cycle. Fertil Steril 91:522-527

Elliott K, Cable N, Reilly T (2005) Does oral contraceptive use affect maximum force production in women? Br J Sports Med 39:15-19

Elliott-Sale KJ et al (2013) Examining the role of oral contraceptive users as an experimental and/or control group in athletic performance studies. Contraception 88:408-412

Erskine RM, Morse CI, Day SH, Williams AG, Onambele-Pearson GL (2014) The human patellar tendon moment arm assessed in vivo using dual-energy X-ray absorptiometry. J Biomech 47:1294-1298

Fleischman DS, Navarrete CD, Fessler DM (2010) Oral contraceptives suppress ovarian hormone production. Psychol Sci 21:750-752

Hansen M et al (2009a) Effect of estrogen on tendon collagen synthesis, tendon structural characteristics, and biomechanical properties in postmenopausal women. J Appl Physiol 106:1385-1393

Hansen M et al (2009b) Effect of administration of oral contraceptives in vivo on collagen synthesis in tendon and muscle connective tissue in young women. J Appl Physiol 106:1435-1443

Hansen $M$ et al (2013) Impact of oral contraceptive use and menstrual phases on patellar tendon morphology, biochemical composition, and biomechanical properties in female athletes. J Appl Physiol 114:998-1008

Heald A, Kaushal K, Anderson S, Redpath M, Durrington PN, Selby PL, Gibson MJ (2005) Effects of hormone replacement therapy on insulin-like growth factor (IGF)-I, IGF-II and IGF binding protein (IGFBP)-1 to IGFBP-4: implications for cardiovascular risk. Gynecol Endocrinol 20:176-182

Hicks K, Onambele Pearson G, Winwood K, Morse C (2013) Gender differences in fascicular lengthening during eccentric contractions: the role of the patella tendon stiffness. Acta Physiol 209:235-244

Hicks K, Onambélé G, Winwood K, Morse C (2016) Muscle damage following maximal eccentric knee extensions in males and females. PloS one 11:e0150848

Hoffman BW, Cresswell AG, Carroll TJ, Lichtwark GA (2014) Muscle fascicle strains in human gastrocnemius during backward downhill walking. J Appl Physiol 116:1455-1462

Hyatt J, Clarkson P (1998) Creatine kinase release and clearance using MM variants following repeated bouts of eccentric exercise. Med Sci Sports Exerc 30:1059-1065

Jamurtas AZ et al (2005) Comparison between leg and arm eccentric exercises of the same relative intensity on indices of muscle damage. Eur J Appl Physiol 95:179-185
Joyce S, Sabapathy S, Bulmer AC, Minahan C (2014) The effect of prior eccentric exercise on heavy-intensity cycling: the role of gender and oral contraceptives. Eur J Appl Physiol 114:1-9

Landgren B-M, Undén A-L, Diczfalusy E (1980) Hormonal profile of the cycle in 68 normally menstruating women. Acta Endocrinol 94:89-98

Legro RS et al (2008) Effects of continuous versus cyclical oral contraception: a randomized controlled trial. J Clin Endocr Metab 93:420-429

Lippold O (1952) The relation between integrated action potentials in a human muscle and its isometric tension. J Physiol 117:492

MacNeil LG, Baker SK, Stevic I, Tarnopolsky MA (2011) 17 $\beta$-estradiol attenuates exercise-induced neutrophil infiltration in men. Am J Physiol Regul Integr Comp Physiol 300:R1443-R1451

Minahan C, Joyce S, Bulmer AC, Cronin N, Sabapathy S (2015) The influence of estradiol on muscle damage and leg strength after intense eccentric exercise. Eur J Appl Physiol 115:1493-1500

O'Brien TD, Reeves ND, Baltzopoulos V, Jones DA, Maganaris CN (2010) Mechanical properties of the patellar tendon in adults and children. J Biomech 43:1190-1195

Olesen JL, Heinemeier KM, Haddad F, Langberg H, Flyvbjerg A, Kjær M, Baldwin KM (2006) Expression of insulin-like growth factor I, insulin-like growth factor binding proteins, and collagen mRNA in mechanically loaded plantaris tendon. J Appl Physiol 101:183-188

Onambélé GNL, Burgess K, Pearson SJ (2007) Gender-specific in vivo measurement of the structural and mechanical properties of the human patellar tendon. J Orthopaed Res 25:1635-1642

Roberts TJ, Azizi E (2010) The series-elastic shock absorber: tendons attenuate muscle power during eccentric actions. J Appl Physiol 109:396-404

Roberts TJ, Konow N (2013) How tendons buffer energy dissipation by muscle. Exerc Sport Sci Rev 41:186-193

Roth SM, Gajdosik R, Ruby B (2001) Effects of circulating estradiol on exercise-induced creatine kinase activity. J Exerc Physiol 4:10-17

Savage KJ, Clarkson PM (2002) Oral contraceptive use and exerciseinduced muscle damage and recovery. Contraception 66:67-71

Sewright KA, Hubal MJ, Kearns A, Holbrook MT, Clarkson PM (2008) Sex differences in response to maximal eccentric exercise. Med Sci Sports Exerc 40:242

Silvestri A et al (2003) Increased levels of C-reactive protein after oral hormone replacement therapy may not be related to an increased inflammatory response. Circulation 107:3165-3169

Sneader W (2005) Hormone analogues. In: Hoboken NJ (ed) Drug discovery: a history, John Wiley, pp 188-225

Thompson HS, Hyatt J-P, De Souza MJ, Clarkson PM (1997) The effects of oral contraceptives on delayed onset muscle soreness following exercise. Contraception 56:59-65

Van Heusden A, Fauser B (2002) Residual ovarian activity during oral steroid contraception. Hum Reprod Update 8:345-358

Walsh L, Hesse C, Morgan D, Proske U (2004) Human forearm position sense after fatigue of elbow flexor muscles. J Physiol 558:705-715

Warren GL, Lowe DA, Armstrong RB (1999) Measurement tools used in the study of eccentric contraction-induced injury. Sports Med 27:43-59

Wiseman H, Quinn P, Halliwell B (1993) Tamoxifen and related compounds decrease membrane fluidity in liposomes: mechanism for the antioxidant action of tamoxifen and relevance to its anticancer and cardioprotective actions? FEBS Lett 330:53-56 